\title{
AN TRAFFIC CONTROL METHOD BASED ON THE REAL-TIME DETECTOR DELAY
}

This paper puts forward a new single intersection control optimization method based on real-time delays by using the new technology of traffic detection. With this method the delay data (including parking delay, acceleration and deceleration delays) of vehicles and speed of the vehicles at the stop bar in every cycle can be detected real-timely. Thus, we can change the cycle length, total split, yellow time and all red time according to the analysis of the reasons of delay changes so as to optimize the traffic signal timing. Compared with traditional methods, this method can effectively deal with the situation that the traffic control scheme does not conform to the actual situation, resulting from the change of the road capacity under the conditions of rain, snow or fog, etc. The good effect can be seen in the example of this research as it effectively reduced delay by $19 \%$ on average.

Keyword: real-time detector delay, traffic control, traffic control results

\section{Introduction}

As there are numerous factors influencing the results of traffic control which can not all be detected, only the parameters such as traffic volumes and the queue length are detected in the classic method, assuming that the parameters such as the road traffic capacity, friction coefficient, vehicle sight distance on road are constant. The classic method can adapt to most weather cases but rain, snow and fog, when the parameters like road friction coefficient are changed, the control optimization system can not be selfadjusted effectively to adapt to the variation of parameters, and so great vehicle delay will be caused. Since the influencing factors can not all be detected, a new single intersection traffic control optimization method is proposed from another standpoint in this paper relying mainly on the traffic control results (delay) of traffic control.

\section{Research Status}

A traffic control theory has developed for nearly one hundred years, and many encouraging achievements have been made. The upgrade of Anchorage Signal System was finished [1], the Adverse Weather Traffic Signal Timing was researched [2, 3, 4, 5], the SCOOT System has been studied [6, 7], and the SCATS System was studied $[8,9]$.The adaptive signal control strategies have been improved endlessly [10].

Despite of lots of achievements gained, the research is all about how to control traffic in accordance with the detected factors such as traffic volume and queue. As for the effect of the control, the system can not detect or evaluate it by itself, let alone changing its strategy according to the effect.

\section{Contents}

\subsection{Basic Principle}

The traditional control method can change a traffic control scheme by detector factors real-timely which influence the traffic control. Due to the diversity of the influencing factors and the real conditions, only the main factors (such as traffic volumes) can be detected. Adaptive control (as shown in Figure 1) is considered to be the most advanced traffic control form, which can only achieve the adoption to input (traffic flow), but can not adjust the control strategy according to the output (mainly delays) appropriately. This paper presents a new idea which detects the control results mainly (as shown in Figure 2). Its research contents include the detection of delay, analysis of the reasons which cause the delay and the adjustment of the control parameters.

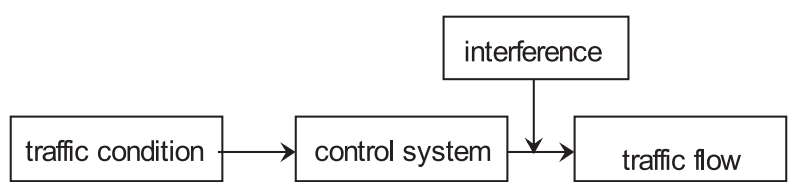

Fig. 1 Schematic diagram of the adaptive control

\footnotetext{
* Bohang Liu ${ }^{1,2}$, Tong Zhang ${ }^{3,4}$, Qingbing Li ${ }^{1,2}$, Lijuan Wang ${ }^{1,2}$

${ }^{1}$ School of Traffic and Transportation, Shijiazhuang Railway Institute, Shijiazhuang, Hebei, China, E-mail: Liubohang@126.com

${ }^{2}$ Traffic Safety and Control Lab of Hebei Province, Shijiazhuang, Hebei, China

${ }^{3}$ The Road Bureau of Sanmenxia, Henna, China

${ }^{4}$ Sanmenxia polytechnic, Henna, China
} 


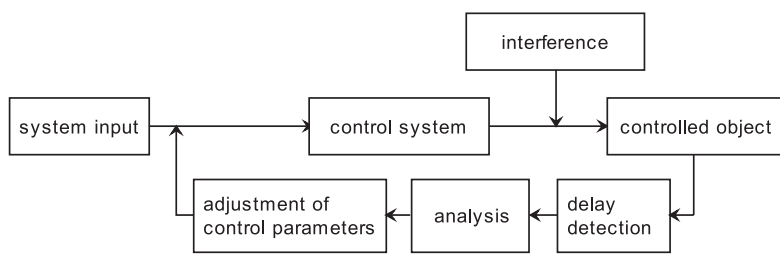

Fig. 2 Schematic diagram of traffic control based on the real-time detector delay

\subsection{Detection of delay}

The delay caused by control can be calculated with the following equation (1) according to the classic method.

$$
d=d 1+d 2+d 3
$$

In which, $\mathrm{d}$ is the delay caused by signal control, $d 1$ is the average delay, $d 2$ is the stochastic additional delay, $d 3$ is the additional delay caused by the queuing of previous vehicles.

Normally, no previous vehicles should exist when vehicles arrive at the signal intersection, that is, the additional delay caused by the queuing of previous vehicles shouldn't exist. Thus the delay in every lane when designing an intersection can be calculated with the following equations:

$$
\begin{aligned}
& d=d_{1}+d_{2} \\
& d_{1}=0.5 C(1-\lambda)^{2} / 1-\min [1, x] \lambda \\
& d_{2}=900 T\left[(x-1)+\sqrt{(x-1)^{2}+T \frac{8 e X}{C A P}}\right]
\end{aligned}
$$

Where,

$C$ - the time of cycle;

$\lambda \quad$ - split of the calculated lane;

$x \quad$ - saturation of the calculated lane;

$C A P$ - capacity of the calculated lane $(\mathrm{pcu} / \mathrm{h})$;

$t$ - time of duration of the analyzed interval, taken as $0.25 \mathrm{~h}$;

$e \quad$ - correction coefficient of the signal control in a single intersection;

The system delay is detected real-timely in this paper based on video traffic detector system, and Fig. 1 illustrates the principle:

As shown in Fig. 1, vehicles generally experience four steps and cause three delays in the signal intersection, the four steps are decelerating, parking, accelerating and leaving at a normal speed; the three delays are deceleration delay, parking delay and acceleration delay. Vehicles in the areas of D1 are decelerating and D2 are parking and D3 are accelerating. At present, the technology of traffic video detection is getting more mature with each passing day [11,12], which can track trajectory and real-time status of a single vehicle. The main steps are extracting background, identifying characteristics of a vehicle's contour and tracking the feature points and so on. The commonly used methods are Kalman filtering, hidden Markov filtering, extended Kalman filtering, Particle swarm filtering and others. As introduced in reference 13, the accuracy of tracking can reach $98 \%$ in the case of no block and $92 \%$ in the case of block. Therefore, the average delay is put forward in this paper on that basis, which can be expressed by equation (5):

$$
d=\sum_{i=1}^{n} d_{i} / n=\sum_{i=1}^{n}\left(t_{i 1}-t_{i 2}\right) / n
$$

In which,

$d \quad$ - the average delay of vehicles at the signal intersection

$d_{i} \quad$ - the actual delay of every vehicle;

$t_{i 1} \quad$ - time for the vehicle to travel from a picture edge of video detection to the stop bar;

$t_{i 2}$ - time for the vehicle to travel from a picture edge of video detector to the stop bar under ideal conditions;

$n$ - number of vehicles;

The headway used in calculating road capacity can be calculated with the equation below:

$$
t_{i}=\sum_{j=3}^{n-m-1} t_{j} /(n-3-m)
$$

Where,

$t_{i} \quad$ - the average time for the other vehicles except the first two vehicles to pass; that is the headway

$t_{j} \quad$ - the headway between vehicles $J$ and $J+1$ in this lane and this cycle;

$n$ - the number of vehicles passing a lane in one cycle;

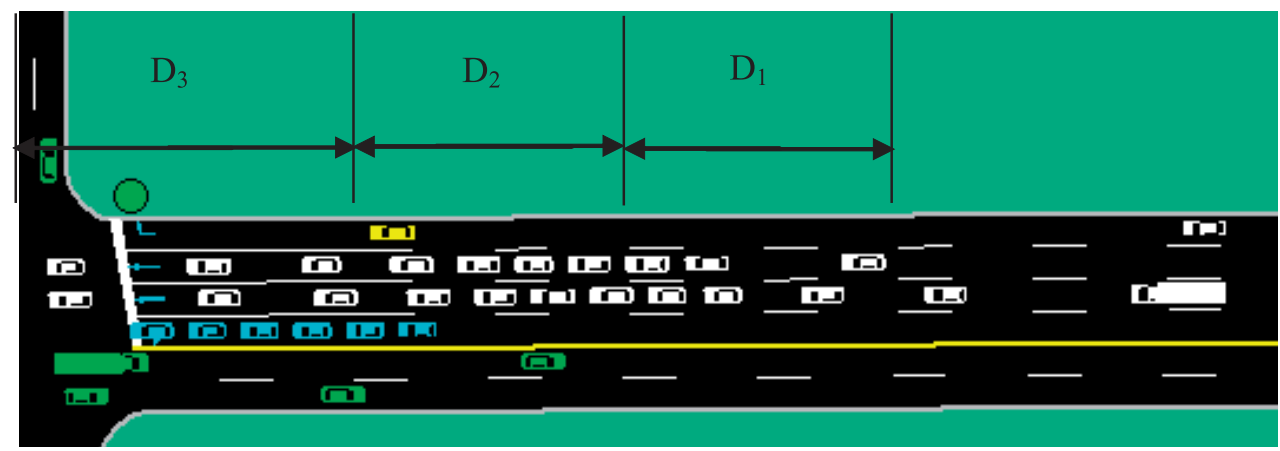

Fig. 3 Principle diagram of delay generation 
$m$ - the number of vehicles passing separately at the later period of the green light; the headway can't be used in calculating road capacity in this period;

The speed of vehicles passing the stop bar can be achieved by calculating the average while the acceleration and deceleration parameters can be achieved by tracking.

\subsection{Analysis of the causes for delay}

The delay changes can result from two reasons, (1) the traffic volumes changes; (2) changes of other factors.

It is the most common situation that the traffic delay changes due to the change of traffic volumes, which can be dealt with by classic adaptive control. There are many processing methods, like TRB method, the TRRL method and the United States HCM manual method. The algorithm is very mature and widely used and we don't list them individually.

It is indicated that there are other factors affecting traffic operation in the intersection, if the traffic volumes doesn't change much but the detected delay changes dramatically. Usually they are rain, snow or fog, and these adverse weathers can be considered as having effect on the capacity of entrance lanes vehicle speed and acceleration.

The classical control method is used in the first situation, therefore only the second situation is discussed in the content of 3.4 and 3.5.

\subsection{Analysis of signal timing Principle}

Figure 4 illustrates the adjustment principle of the traffic control system mentioned in 3.3 , which is under the second condition. Namely, if the detected real-time delay increases, in most cases, it is because the weather factors make the road friction coefficient and sight distance of the driver decrease, thus the phase intervals and the cycle are impacted.

(1) Phase Change Interval

$$
y+r=\delta+\frac{v}{2 a+G}+\frac{w+L}{v}
$$

Where $Y+A R=$ Sum of the yellow and all red time intervals $T=$ perception/reaction time of driver in seconds (typically taken as 1.0 second) $V=$ approach speed in feet per second $a=$ deceleration rate $t$ per second $w=$ Width of intersection $L=$ length of vehicle $g r=$ approach grade, percent of grade divided by 100 (add for up-grade and subtract for downgrade)

Because the length of the intersection is not changed and the vehicle speed decreases, the phase change interval will be increased.

(2) Cycle:

$$
c=\frac{1.5 L+5}{1-Y}
$$

Where, $c$ is the signal cycle; $L$ is the lost time; and $Y$ is the saturation;

The equation to calculate the lost time is as follows:

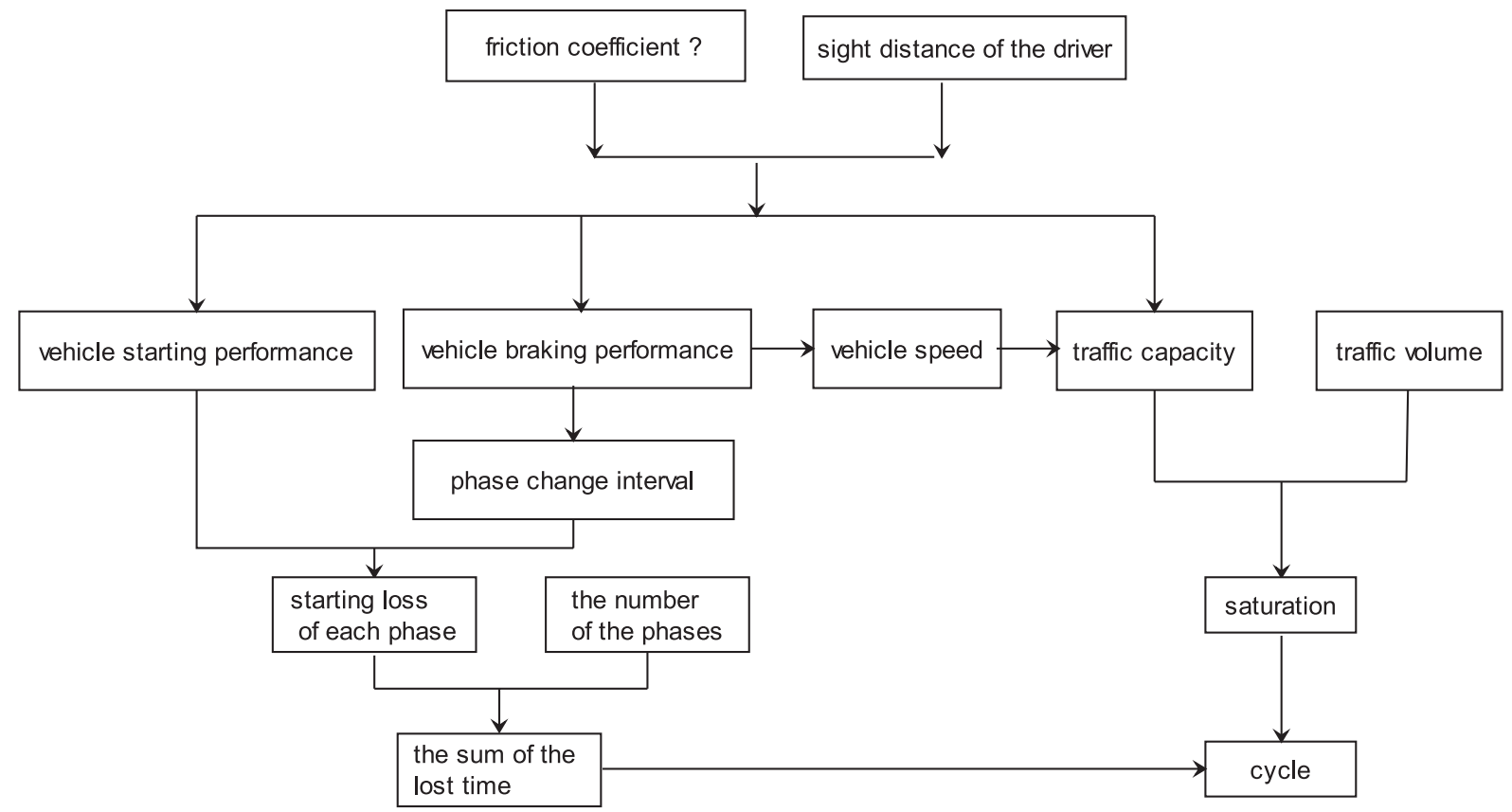

Fig. 4 Relationship between friction coefficient and signal timing parameters 


$$
L=\sum(l+I-A)
$$

In the equation: $L$ is total lost time in one cycle (s); $l$ is startup lost time; $I$ is the phase change interval; and $A$ is the yellow time. A

$\mathrm{Y}$ is the accumulation of each phase saturation;

The others are the same as above

The cycle should be adjusted to bigger due to the increased phase change interval.

\subsection{Adjustment of Control Parameters}

According to the above analysis, the adjustment plan of the Interval time of the green and the cycle can be confirmed when we can identify when the detected real-time delay increases.

After the timing scheme has been recounted as above, it will be applied in the intersection control and the delay of the new scheme will be detected. The detected delay will decrease in most cases. If the delay increases or if there is no obvious change it means that the analysis of the cause of delays is wrong. The vehicle delay variation may be caused by a pedestrian or a non-motor vehicle. It should be re-analyzed and re-timed.

\section{Case Analysis}

One intersection in Shijiazhuang City of China was taken as an example to be analyzed. Traffic flow in this intersection was stable in the experiment, and the basic situation of traffic flow in each entrance is shown in Table 2. In the morning of the experiment the weather was normal, the pavement was dry, the pavement friction coefficient was 0.8 and the normal scheme was used in traffic signal, as shown in Table 3. After snow in the afternoon, the traffic control departments used snow melting agents in the trunk road (Yuhua Road) and the road friction coefficient was 0.6 , while the secondary road (Tiyu Street) was not processed and the road friction coefficient was 0.4 . The system used the algorithm presented in this paper. Several cycles detected showed that after the snow, with a similar number of vehicles, the real-time detection of the vehicles' delay increased. Judging that the road capacity and the speed of vehicles may change, the system calculated the headway and the speed of the vehicles at the stop bar. The new traffic signal timing programs are generated after a re-calculated cycle, total split, the interval of yellow time and green time as shown in Table 3. Table 4 demonstrates effect comparisons among the cases. Obviously, the delay caused by the scheme offered in this paper is shorter than using conventional timing scheme under the conditions of snow. The experiment of the timing scheme adjustment is manual due to limited experimental conditions.

Basic situation of the intersection

Table 2

\begin{tabular}{|c|c|c|c|c|c|}
\hline Entrances & $\begin{array}{c}\text { East } \\
\text { entrances }\end{array}$ & $\begin{array}{c}\text { West } \\
\text { entrances }\end{array}$ & $\begin{array}{c}\text { South } \\
\text { entrances }\end{array}$ & $\begin{array}{c}\text { North } \\
\text { entrances }\end{array}$ & Sum \\
\hline Volume of traffic & 2160 & 2090 & 452 & 502 & 5204 \\
\hline Number of lanes & 4 & 4 & 2 & 2 & 12 \\
\hline Capability & 4187.00 & 4215.00 & 2178.00 & 2098.00 & 12678.00 \\
\hline $\mathrm{v} / \mathrm{c}$ & 0.52 & 0.50 & 0.21 & 0.24 & - \\
\hline
\end{tabular}

\begin{tabular}{|c|c|c|c|c|}
\hline \multirow{6}{*}{1} & \multirow{6}{*}{$\begin{array}{l}\text { Interval } \\
\text { time of the } \\
\text { green }\end{array}$} & \multirow{3}{*}{ First } & Detected headway increases & Interval increases \\
\hline & & & Detected headway decreases & Interval decreases \\
\hline & & & Not the above situations & No adjustment \\
\hline & & \multirow{3}{*}{ Second } & Vehicle delay in the intersection increases after the last adjustment & Adjustment in the opposite direction \\
\hline & & & Vehicle delay decreases in the intersection after the last adjustment & Adjustment in the same direction \\
\hline & & & Vehicle delay increases in the intersection after the adjustment in both directions & Stop adjustment \\
\hline \multirow{6}{*}{2} & \multirow{6}{*}{ Cycle } & \multirow{3}{*}{ First } & The headway or the traffic volume increases & Cycle increases \\
\hline & & & The headway or the traffic volume decreases & Cycle decreases \\
\hline & & & Not the above situations & No adjustment \\
\hline & & \multirow{3}{*}{ Second } & Vehicle delay in the intersection increases after the last adjustment & Adjustment in the opposite direction \\
\hline & & & Vehicle delay decreases in the intersection after the last adjustment & Adjustment in the same direction \\
\hline & & & Vehicle delay decreases in both directions & Stop adjustment \\
\hline
\end{tabular}

Signal timing scheme

Table 3

\begin{tabular}{|c|c|c|c|c|c|c|c|}
\hline Entrances & $\begin{array}{c}\text { First phase time (traffic } \\
\text { in east and west) }\end{array}$ & Yellow time & All red time & $\begin{array}{c}\text { Second phase (traffic } \\
\text { in south and north) }\end{array}$ & Yellow time & All red time & Cycle \\
\hline $\begin{array}{c}\text { Under the normal } \\
\text { timing scheme }\end{array}$ & 35 & 3 & 0 & 16 & 3 & 0 & 57 \\
\hline $\begin{array}{c}\text { Timing scheme under } \\
\text { the condition of snow }\end{array}$ & 89 & 3 & 1 & 41 & 3 & 1 & 138 \\
\hline
\end{tabular}


Delay comparison of the intersection

Table 4

\begin{tabular}{|c|c|c|c|c|c|}
\hline Delay per vehicle & East entrances & West entrances & South entrances & North entrances & Average \\
\hline Under normal condition & 8.1 & 7.2 & 14.7 & 17.9 & 9.4 \\
\hline Normal scheme under the condition of snow & 23.6 & 20.2 & 56.4 & 45.2 & 27.4 \\
\hline This scheme under the condition of snow & 19.9 & 16.1 & 25.9 & 27.6 & 22.3 \\
\hline
\end{tabular}

\section{Conclusion}

This paper puts forward a new single intersection control optimization method based on real-time delays, by using the new technology of traffic detection. With this method the delay data (including parking delay, acceleration and deceleration delay) of vehicles and the speed of the vehicles at the stop bar in every cycle can be detected real-timely. We can thus change the cycle length, total split, yellow time and all red time according to the analysis of the reasons of delay changes, so as to optimize the traffic signal timing. This method breaks away with the traditional one which detects the influencing factors one by one. It detects the control results directly and analyzes the reasons of how they come into being. Compared with traditional methods, this method can effectively deal with the situation that the traffic control scheme does not conform to the actual situation resulting from the change of the road capacity under the conditions of rain, snow, fog, etc. The good effect can be seen in the example of this research as it effectively reduced delay by $19 \%$ on average.

\section{References}

[1] LOCHMUELLER, B. and Associates: Anchorage Signal System Upgrade-Final report. 1995

[2] MAKI, P. J.: Adverse Weather Traffic Signal Timing, P.E. $69^{\text {th }}$ Annual Meeting of the ITE, Las Vegas, 1999

[3] PARSONSON, P. S.: Flashing Operation and Signal Timing for Adverse Weather, NCHRP Synthesis of Highway Practice, 1992, pp. 46-47.

[4] BOTHA, J. L., KRUSE, T. R.: Flow Rates at Signalized Intersections under Cold Winter Conditions, J. of Transportation Engineering, 1992, pp. 439-450.

[5] GILLAM, W. J., WITHILL, R. A.: UTC and Inclement Weather Conditions, IEE Conference, 1992, pp. 85-88

[6] HUNT, P. B., ROBERTSON, D. I., BRETHERTON, R. D., WINTON R. I.: SCOOT-A Traffic Responsive Method of Coordinating Signals; TRRL Report LR1014, Crowthorne, 1981

[7] HANSEN, B., MARTIN, P., PERRIN, J.: SCOOT Real-Time Adaptive Control in a CORSIM Simulation Environment, Transportation Research Board (TRB), 2000 Annual Conference, Washington, D. C., January 2000 (accepted for Publication in TRR)

[8] LOWRIE, P. R.: SCATS, Sydney Co-ordinated Adaptive Traffic System, A Traffic Responsive Method of Controlling Urban Traffic, Roads and Traffic Authority Sydney, NSW, Australia, 1990.

[9] LOWRIE, P. R.: The Sydney Co-ordinated Adaptive Traffic System Principles, Methodology Algorithms, Int. conference on Road Traffic Signaling, London, 1982, pp. 67-70.

[10] GARTNER, N. H., STAMATIADIS, C., TARNOFF, P. J.: Development of Adaptive Signal Control Strategies for Intelligent Transportation Systems: A Multilevel Design, Presented at the $74^{\text {th }}$ Annual meeting of the Transportation Research Board, 1995.

[11] KOLLER D, WEBER J, MALIK J.: Robust Multiple Car Tracking with Occlusion Reasoning, Proc. of European Conference on Computer vision (ECCV), Berlin-Heidelberg; Springer-Verlag, 1994, pp. 189-196

[12] ROMBERG, J. K, CHOI, H, BARANIUK, R. G.: Bayesian Tree-structured Image Modeling Using Wavelet-domain Hidden Markov Models, IEEE Transactions on Image Processing, 2001, pp. 1056-1068. 